\title{
Further evidence for the role of magnetotail current shape in substorm initiation
}

\author{
M. Kubyshkina ${ }^{*}$, N. Tsyganenko ${ }^{1}$, V. Semenov ${ }^{1}$, D. Kubyshkina ${ }^{1}$, N. Partamies² and E. Gordeev
}

\begin{abstract}
Substorm initiation still remains an unsolved problem, even though there is a consensus among most researchers that its main stages include dayside reconnection and substorm expansion. Dayside reconnection results in magnetotail flux buildup to a certain critical level, after which [or after the interplanetary magnetic field (IMF) Bz turns northward] the substorm expansion begins. One problem with the above scenario is that the critical amount of magnetic flux differs from one substorm to another, and not every northward turning of the IMF Bz triggers a substorm. We suggest that an important factor in substorm dynamics may be the variable shape and alignment of the magnetospheric tail current sheet, which bends and warps in response to diurnal/seasonal changes of the Earth's dipole tilt angle and also in response to more rapid changes of the solar wind flow direction. Both of these factors may be important, if one assumes that the deformed current sheet becomes unstable at lower values of the tail lobe magnetic flux/pressure than the planar sheet. To investigate this idea, we examined large multi-year sets of THEMIS, Cluster, and Geotail data and established a relationship between the tail lobe Bx and the dipole tilt angle. Further examinations of substorm events during 2005-2010 supported the hypothesis that their probability and intensity indeed depended on the concurrent values of the tilt angle.
\end{abstract}

Keywords: Magnetospheric substorm; Tail current sheet; Empirical modeling

\section{Background}

Substorm initiation is a controversial topic in magnetospheric physics, especially because it is complicated by the variability of both magnetotail/ionosphere substorm dynamics and related solar wind parameters. In some cases, a relatively small amount of solar wind energy input may cause substorm onset, while in other cases, a larger input produces no effect at all. This implies that (1) not only are the solar wind (SW) and interplanetary magnetic field (IMF) important factors but also is the magnetotail configuration important as well and (2) there exist additional characteristics of the SW that may play an important role in substorm onset. In this paper, we explore one such additional factor, namely, the shape of the current sheet, which is subject to periodic deformations due to the regular variations of the dipole tilt angle and more irregular changes of the SW direction that result in windsock deflection of the nightside magnetosphere.

*Correspondence: kubysh@geo.phys.spbu.ru

1 Saint Petersburg State University, St. Petersburg, Russia

Full list of author information is available at the end of the article
A possible relation between the shape of the tail current sheet and its dynamics has been suggested in earlier work by Kivelson and Hughes (1990). The authors introduced the idea of the possible influence of that factor on the substorm instability threshold, i.e., a bent magnetotail configuration with larger curvature of the field lines is more favorable for the formation of a new near-Earth neutral line and thus results in substorm onset triggered by a smaller energy input from the SW. Therefore, a stronger tail bending would result in a larger number of smaller substorms, which break after less magnetic flux increase during the growth phase. Smaller flux increase during substorms would produce a statistical effect of a lower averaged lobe magnetic field during the periods with larger dipole tilts, and this effect may be deduced from many years of spacecraft observations in the magnetotail. However, this idea was not validated at that time by in situ observations probably because of the lack of spacecraft data. In a more recent work, Partamies et al. (2013) performed a statistical analysis of the substorm occurrence rate in different seasons and reported that substorms occur more frequently not around the equinox

\section{Springer}

(c) 2015 Kubyshkina et al. Open Access This article is distributed under the terms of the Creative Commons Attribution 4.0 International License (http://creativecommons.org/licenses/by/4.0/), which permits unrestricted use, distribution, and reproduction in any medium, provided you give appropriate credit to the original author(s) and the source, provide a link to the Creative Commons license, and indicate if changes were made. 
but during mid-winter months and in August, though the largest negative peak Dst (and largest Kp) fell on April and October. A statistical study by Nowada et al. (2009) showed that auroral electrojet indices reached higher values in the periods with smaller dipole tilt angles and thus, supported the idea by Kivelson and Hughes (1990) that larger number of smaller substorms occur in the periods of maximal dipole tilt. Additional evidence to revise the relationship between the substorm onset and tail configuration was provided by a number of case studies (e.g., Kubyshkina et al. 2011; Sergeev et al. 2011; Panov et al. 2012, Ganushkina et al. 2013), in which substorms were shown to be initiated in a curved magnetotail with excessively (due to additional external tilt) increased plasma sheet bending.

The purpose of this paper is to statistically confirm the influence of tail current configuration on substorm onset by investigating the following phenomena: (1) substorm probability during periods with different dipole tilt angles and SW flow directions, (2) AL index dependence on the current sheet bending, and (3) the magnetotail lobe field magnitude as a function of the dipole tilt angle.

\section{Substorm probability and current sheet bending Data base}

The shape of the magnetospheric current sheet is varying continuously every moment. At close geocentric distances, it is controlled by the orientation of the Earth's magnetic dipole axis and periodically changes in response to the planet's diurnal rotation and its orbital motion around the Sun. The distant current sheet extends antisunward and follows the randomly changing SW direction in a windsock-like manner. If the SW is purely radial (flows in the anti-solar direction) and the Earth's dipole is perpendicular to the ecliptic plane (dipole tilt $\Psi_{\mathrm{GSM}}-$ angle between the $Z_{\mathrm{GSM}}$ axis and Earth's dipole axis-is zero), we expect to have a planar current sheet and northsouth symmetric field lines. The deflection of SW from the radial direction would cause the displacement of the distant current sheet from the equatorial plane in accordance with SW flow. The dipole axis rotation (dipole tilt increase) will move the near-Earth portion of the current sheet from the ecliptic plane to the magnetic equator creating a bent and shifted current sheet at closer equatorial distances. These three configurations are schematically shown in Fig. 1 in the top, middle, and bottom panels. The most recent data-based studies of the global shape of the neutral sheet and its position with respect to the magnetospheric equatorial plane were carried out by Tsyganenko and Fairfield (2004), Tsyganenko and Andreeva (2014), and Tsyganenko et al. (2015). In these works, the observed deformation of the neutral sheet was described by empirical equations representing its average shape in the Geocentric Solar Wind (GSW) coordinate system with

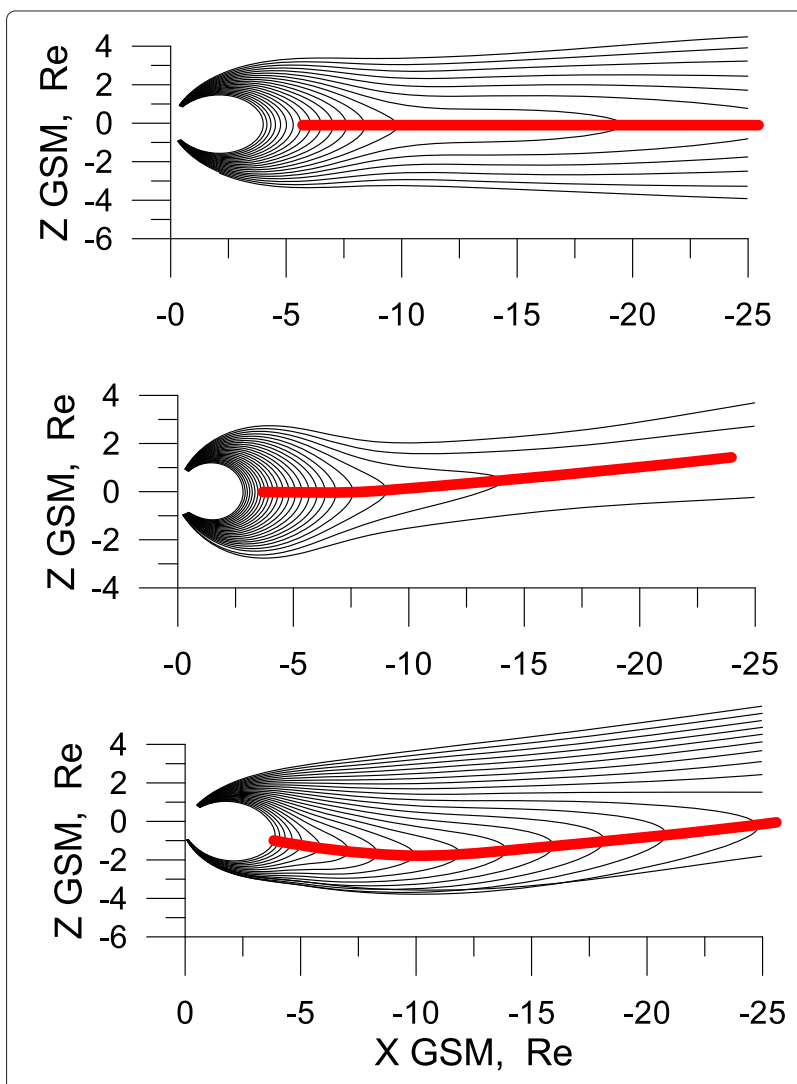

Fig. 1 Top panel: field line configuration for the perpendicular dipole and radial SW flow. Middle panel: the same with non-radial SW (positive V $z_{s w}$ ). Bottom panel: field lines for the negative dipole tilt and non-radial SW flow (positive $V z_{S W}$ )

the $X_{\mathrm{GSW}}$ axis antiparallel to the observed SW flow vector rather than to the Sun-Earth line [as assumed in the standard Geocentric Solar Magnetospheric (GSM) system]. Larger tilt angles result in stronger deformation of the near-Earth current sheet at $r \leq 10-15 R_{\mathrm{E}}$ and in larger shifts from the equatorial plane at greater distances. The effective tilt angle $\Psi_{\mathrm{GSW}}$ (the angle between the $Z_{\mathrm{GSW}}$ axis and Earth's dipole axis) depends not only on the date/UT (which defines the tilt angle $\Psi_{\mathrm{GSM}}$ in the standard solar-magnetospheric coordinate system) but also on the orientation of the SW velocity vector $\mathbf{V}_{\mathrm{SW}}$. The angle $\Psi_{\mathrm{GSW}}$ can be calculated by means of a standard software package GEOPACK-2008, which is available from http:// geo.phys.spbu.ru/ tsyganenko/Geopack-2008.html.

In further analysis, our main interest is focused, however, on the effects of current sheet bending and rotation in the $X Z_{\mathrm{GSM}}$ plane, which may be described with the combined variations of regularly varying standard dipole tilt $\Psi_{\mathrm{GSM}}$ and rapidly changing SW flow direction in the $X Z_{\mathrm{GSM}}$ plane. For that purpose, instead of using the highly variable GSW coordinate system and $\Psi_{\mathrm{GSW}}$ based on the three-component $\mathrm{SW}$ velocity vector, we remained in 
the frame of standard GSM coordinates and introduced the effective tilt $\Psi_{\mathrm{SW}}=\Psi_{\mathrm{GSM}}+\arctan \left(V z_{\mathrm{SW}} / V x_{\mathrm{SW}}\right)$. Here, $V z_{\mathrm{SW}}$ and $V x_{\mathrm{SW}}$ are the SW flow velocity components.

The statistical distribution of the dipole tilt angle $\Psi_{\mathrm{GSM}}$ over a year is illustrated by a histogram in the left panel of Fig. 2, and the data show the numbers of 5-min intervals with $\Psi_{\mathrm{GSM}}$ values falling into consecutive $2^{\circ}$ intervals. The distribution is nearly symmetric with respect to $\Psi_{\mathrm{GSM}}=$ 0 , there are two peaks at $\pm 12^{\circ}-14^{\circ}$, and the values rapidly fall off towards the edges at $\left|\Psi_{\mathrm{GSM}}\right| \approx 33^{\circ}$. The slight asymmetry is due to the ellipticity of the Earth's orbit around the Sun, which results in a somewhat longer (by $\sim 4 \%$ ) total time with positive tilt angles accrued during summer months. The right panel in the same figure presents a similar histogram for the SW flow deflection angle in the $X Z$-plane, which is calculated as $\alpha=$ $\arctan \left(V_{z \mathrm{SW}} / V_{x \mathrm{SW}}\right)$, where $V_{z \mathrm{SW}}$ and $V_{x \mathrm{SW}}$ are the $\mathrm{SW}$ flow velocity components. One can see that the number of 5-min periods with a given flow direction during 1 year (here, 2007) has a very regular, nearly Gaussian distribution, and in most of the 5-min periods the $\alpha$ angle does not exceed $4^{\circ}$. The 5 -min average SW data were downloaded from the OMNIWeb site (http://omniweb.gsfc.nasa.gov/).

To analyze the possible dependence of substorm probability on current sheet bending, we used substorm onsets (beginnings of the expansion phase) identified as abrupt decreases of the magnetic AL index. This method for automatic substorm phase detection has been described in more detail in Juusola et al. (2011) and Partamies et al. (2013). Out of all detected expansion phases, we selected those with AL minima of at least $-50 \mathrm{nT}$. For the Northern Hemisphere, we used THEMIS ground-based observations during 2007-2009, and for the Southern Hemisphere, all available data were employed. The southern $\mathrm{AL}$ index data were downloaded from the virtual magnetic observatory at vmi.gsfc.nasa.gov as prepared by the
University of California Los Angeles (UCLA). As a result, we detected 6091 substorms in the Northern Hemisphere and 2170 substorms in the Southern Hemisphere. The resulting numbers of substorms during the periods with different dipole tilt values $\left(\Psi_{\mathrm{GSM}}\right)$ are given for each hemisphere separately and for both hemispheres in the top row of Fig. 3. All three histograms show distinct minima near the zero tilt, two local maxima around $\left|\Psi_{\mathrm{GSM}}\right| \sim 15^{\circ}$, and gradual decreases towards the ends of the tilt angle range. In general, the distributions are very similar to that shown in the left panel of Fig. 2, but with one important difference; there is a relatively larger number of substorms for $\Psi_{\mathrm{GSM}}<-20^{\circ}$ in the Southern Hemisphere and for $\Psi_{\mathrm{GSM}}>20^{\circ}$ in the Northern Hemisphere. Additionally, note that the total distributions are not quite symmetric, with a larger number of substorms for positive tilt values in the Northern Hemisphere and for negative tilts in the Southern Hemisphere (i.e., corresponding to the local summer periods in each hemisphere).

It is important to note, however, that in order to explore the current sheet bending effects on substorm triggering, one should take into account the changing direction of the SW flow, namely, instead of the standard dipole tilt $\Psi_{\mathrm{GSM}}$, one should use the effective tilt $\Psi_{\mathrm{SW}}=\Psi_{\mathrm{GSM}}+$ $\alpha=\Psi_{\mathrm{GSM}}+\arctan \left(V_{z \mathrm{SW}} / V_{x \mathrm{SW}}\right)$. To that end, we complemented our substorm onset data with OMNI 5-min interplanetary data, i.e., corresponding values of the IMF and $\mathbf{V}_{S W}$ components were ascribed to each substorm onset. The bottom row of Fig. 3 gives the number of substorms in relation to the effective tilt angle $\Psi_{\mathrm{SW}}$. Here, we are primarily interested in the bending and latitudinal deflection of the tail, i.e., the effects in the $X Z_{\mathrm{GSM}}$-plane, such that the $V_{\mathrm{y}}$ component was assumed to be of minor importance. The main features of the distributions in the bottom row remained generally the same as those in the upper row, but the range of values was somewhat larger (up to $\pm 40^{\circ}$ ).
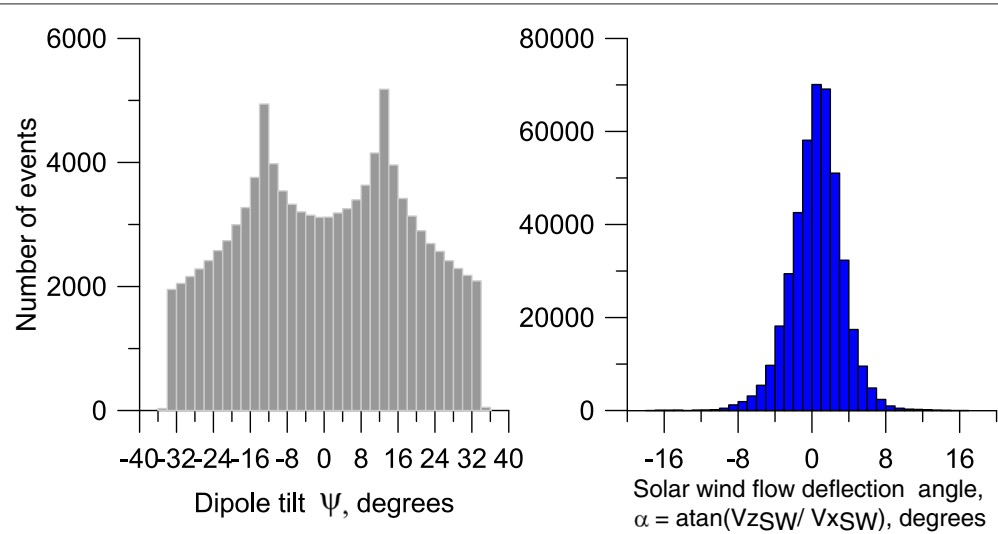

Fig. 2 Left panel: number of 5-min periods with a given dipole tilt $\Psi_{G S M}$ during one year. Right panel: number of 5-min periods with a given SW flow deflection angle from the Sun-Earth line $\alpha=\operatorname{atan}\left(V_{z S W} / V_{x S W}\right)$ during 1 year 

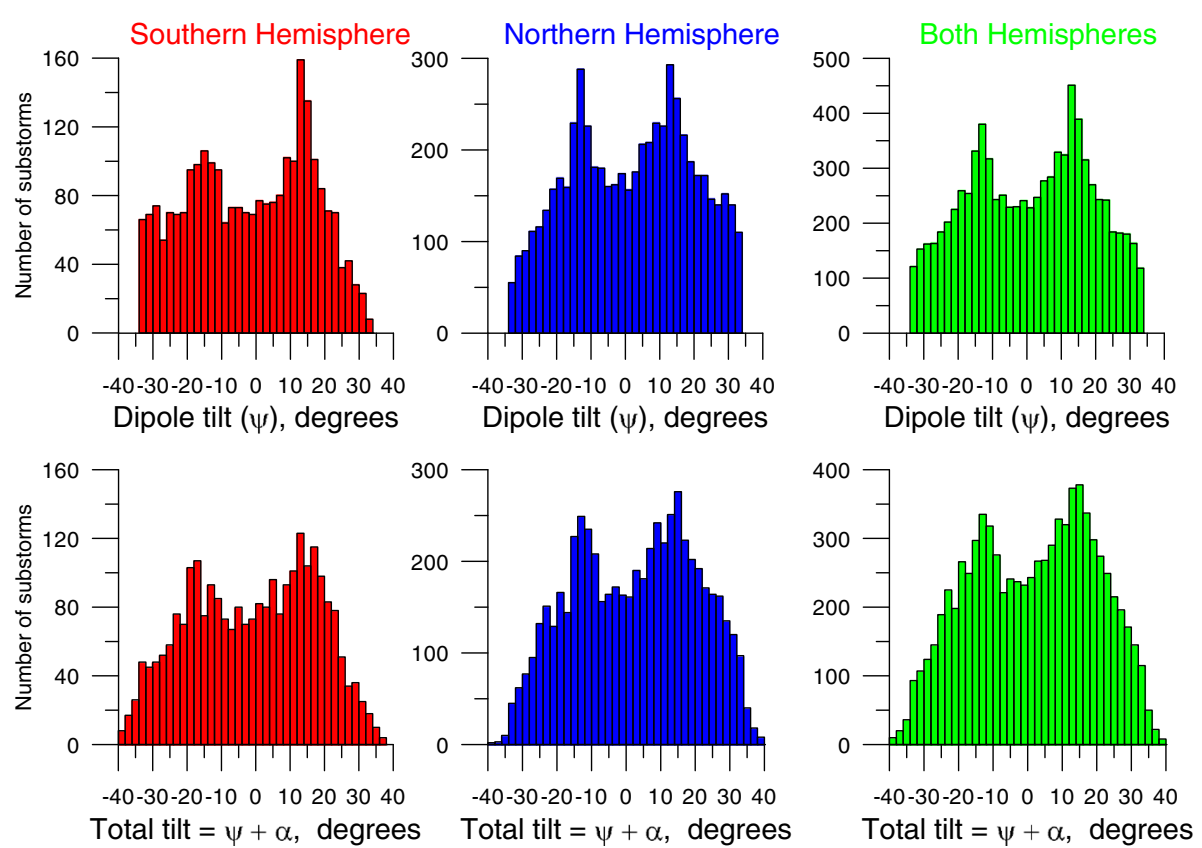

Fig. 3 Number of substorms depending on the dipole tilt $\Psi_{G S M}$ (top row) and on total tilt $\left(\Psi_{G S M}+\alpha\right)$ (bottom row)

\section{Statistical results}

To analyze the substorm probability, we combined the data from both hemispheres and then excluded events from the Northern Hemisphere substorm list that had onsets that were simultaneous (within $\pm 5 \mathrm{~min}$ ) with those detected in the Southern Hemisphere in order to avoid duplications. After that, we normalized the substorm numbers within each bin of the tilt angle by the corresponding values of its occurrence frequency shown in the left panel of Fig. 1. The normalized values are shown in Fig. 4.

A clear increase of the substorm probability with growing tilt can be readily seen for all tilt values, except in the $\pm 24^{\circ}-32^{\circ}$ interval, but those values are offset by a steep increase in the next interval $\pm 32^{\circ}-40^{\circ}$, so that by averaging over these two bins (with a relatively small total number of substorms, see Fig. 3), this again results in an increase of the probability. However, for the negative tilts, the probability values are smaller, which is apparently a result of the more than twice lower number of events from the Southern Hemisphere. On the basis of these data, we conclude that during periods with larger dipole tilt angles, substorm onsets occur with a larger probability than under smaller tilt angles.

Another way to reveal the dependence of substorm occurrence on the effective tilt value is to check whether the SW deflection is more effective when it increases the absolute value of the dipole tilt, i.e., when it makes the magnetotail current sheet more curved. To quantitatively evaluate this possibility, we introduced the function
$\Psi_{\text {norm }}=\Psi_{\mathrm{GSM}} \cdot \operatorname{sign}(\alpha)$, where $\Psi_{\mathrm{GSM}}$ is the standard dipole tilt in the GSM coordinate system and $\alpha$ is the SW velocity inclination in the $X Z_{\mathrm{GSM}}$-plane $\left(\alpha=\arctan \left(V z_{\mathrm{SW}} / V x_{\mathrm{SW}}\right)\right)$. Then, positive values of $\Psi_{\text {norm }}$ would correspond to the same sign of the dipole tilt and the additional tilt due to the SW deflection, while its negative values would correspond to a decrease of effective tilt and, hence, a smaller current sheet curvature. By normalizing $\Psi_{\mathrm{GSM}}$ with $\operatorname{sign}(\alpha)$, we kept the $\Psi_{\text {norm }}$ values within the standard range of $\pm 33^{\circ}$. The resulting histogram of the substorm numbers as a function of $\Psi_{\text {norm }}$ is shown in Fig. 5, where blue (red) columns and blue (red) numbers correspond to the number of substorms for negative (positive) values of $\Psi_{\text {norm}}$. The effect was rather distinct, and all the red columns were $10-20 \%$ higher than the blue ones. The only bin with two equal numbers was the one for the smallest tilt angle range, which was quite naturally expected. This result can be explained if we agree that the substorm initiating instability depends on the total tilt (or degree of current sheet bending). Thus, the already loaded magnetic flux, which is not large enough for a substorm to start under the currently existing dipole tilt, may become efficient after the tilt is further increased by a sudden deflection of the SW in a favorable direction.

The same arguments can also be applied to periods of increasing or decreasing dipole tilt. Then, substorms would preferably start during periods with steadily increasing dipole tilt, while decreasing tilt would result in delayed substorm onsets, even under constant energy loading. Using our data from the Northern Hemisphere 


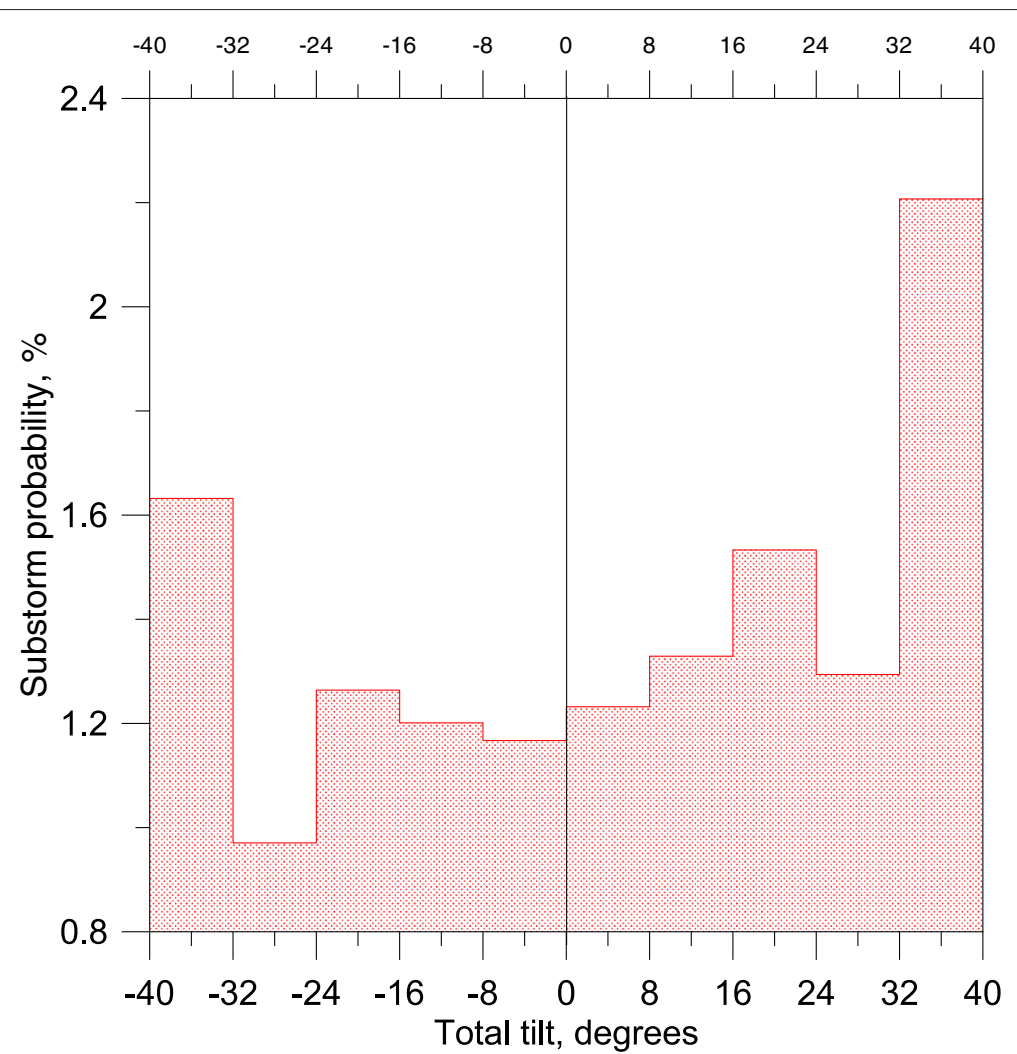

Fig. 4 Substorm probability as a function of the total tilt

(with more regular observations), we compared the number of substorms that occurred during the periods with decreasing and increasing dipole tilt and found that, indeed, the difference does exist, though it is not large, i.e., a little less than $10 \%$ (2917 events with decreasing tilt versus 3173 during tilt increase). To further investigate our conclusion, we performed an identical analysis for the periods of steady magnetospheric convection (SMC) events, when no substorm instability was initiated and substorm expansion did not take place, in spite of a continuous loading. We used a similar data base, which was produced by Kissinger et al. (2011) based on auroral electrojet indices and visual inspection of data, and selected the SMC periods from the same time interval from 2005 through 2007. The obtained result was opposite to that for the substorm events; specifically, we found that about $20 \%$ more SMC events started during the periods of decreasing tilt (218 versus 175 ). One of the many possible reasons for this finding may be, for example, the growth of the substorm instability threshold due to the constantly decreasing tilt.

To summarize, we conclude that during the periods with larger current sheet bending and deflection from the SunEarth line, the number of substorms is larger than during the periods with a more symmetric plasma sheet. This can be seen best in the events when fast SW deflections increase the existing dipole tilt, thus, leading to premature substorm onset. In such situations, the difference from the opposite case (when the SW deflections decrease the effective dipole tilt) may be quite significant, with as much as a $10-20 \%$ increase in the number of substorm onsets.

\section{Substorm intensity and current sheet bending}

Using the above described data base, we also explored if the substorms that started during the periods of strongest tail current bending were initiated after smaller energy loading and, hence, resulted in smaller AL variations. The results of our analysis are illustrated in Fig. 6. The top left panel shows a bar diagram where the horizontal axis represents peak AL values for all the events in our substorm list, and the vertical size of the bars displays the corresponding values of the dipole tilt angle (negative-down, positive-up). It is important to emphasize that we plotted standard tilt angles in this diagram, and even though the correlation between the tilt and AL shows through, it does not seem to be clear enough, especially around the smallest $\mathrm{AL}$ values. The next diagram in the top right panel of the Fig. 6 shows the same data, but with the effective tilt values $\Psi_{\mathrm{SW}}$ plotted along the vertical axis. Here, the correlation appears to be much better, i.e., about $96 \%$ of all 


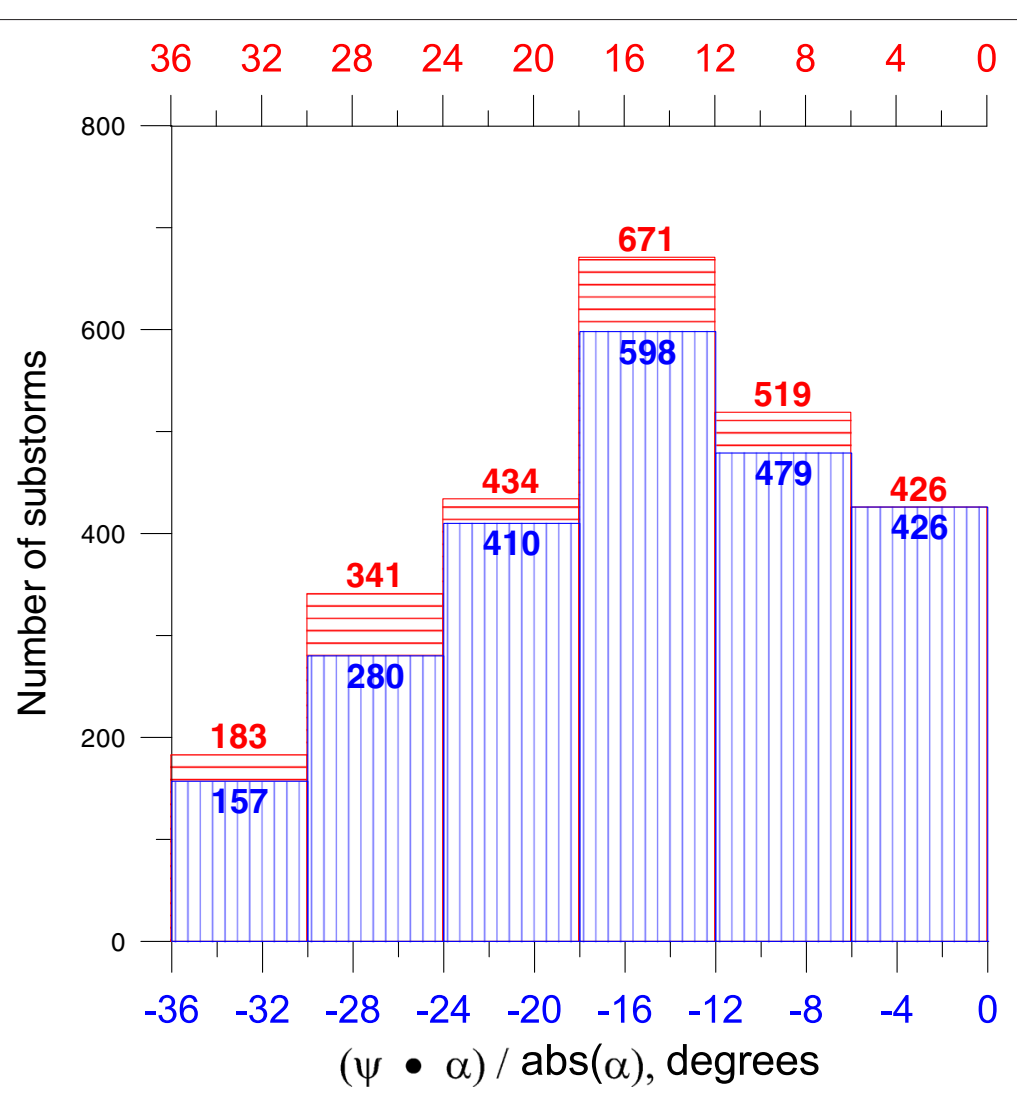

Fig. 5 Number of substorms observed for a given dipole tilt. Blue columns correspond to the situation when the SW flow was decreasing the tilt, and red columns give the substorm numbers when the SW flow was increasing the dipole tilt

substorms lie within the triangle delimited by the red dotted line, so that no intense substorms occur for the largest $\Psi_{\mathrm{SW}}$ values. This tendency is even more pronounced in the bottom left diagram with the products $\left(\Psi_{\mathrm{SW}} \cdot \alpha\right)$ plotted along the vertical axis (note the expanded scale), which further emphasizes the importance of taking into account the SW direction.

Still there is a group of intense substorms with $\mathrm{AL}$ values less than $-600 \mathrm{nT}$, and these occurred in situations with large (more than $20^{\circ}$ ) tilts. We examined the SW parameters for these substorms and found that the majority of them corresponded to high-speed SW intervals. The specific nature of SW-magnetosphere coupling during such periods of fast SW was previously reported, for example, in Partamies et al. (2009). This fact is illustrated in the bottom right panel, where all the events are divided into two groups with SW speeds larger (black) and smaller (red) than $500 \mathrm{~km} / \mathrm{s}$. One can see that most of the intense substorms corresponded to the high-speed SW.

Finally, we conclude that the most intense substorms usually happen during the periods with smaller effective tilts, because the symmetric magnetotail configurations with a planar current sheet more effectively accumulate and store the lobe magnetic flux. On the contrary, the substorms that happen in a curved current sheet have lower intensity, since the curved current sheet is less stable and breaks after smaller energy input.

\section{Lobe magnetic field and current sheet bending}

If we admit, following the arguments in the previous sections, that substorms are set off after a lower energy input during periods of increased dipole tilt, one would expect the average magnetic flux stored in the magnetospheric tail to be lower during the periods with larger tilts, in comparison with the periods with small or zero tilts. Even though we cannot directly estimate the magnetic flux, the lobe magnetic field $B_{\text {lobe }}$ can serve as a good proxy for the flux. Unlike the latter, $B_{\text {lobe }}$ is directly measured by spacecraft, and huge amounts of data have been accumulated over the last few decades. By taking advantage of these developments, we conducted a statistical study to evaluate the possible relation of the lobe field and the effective tilt $\Psi_{\mathrm{GSW}}$ (referred as $\Psi$ throughout this section), which represents the extent of the current sheet 

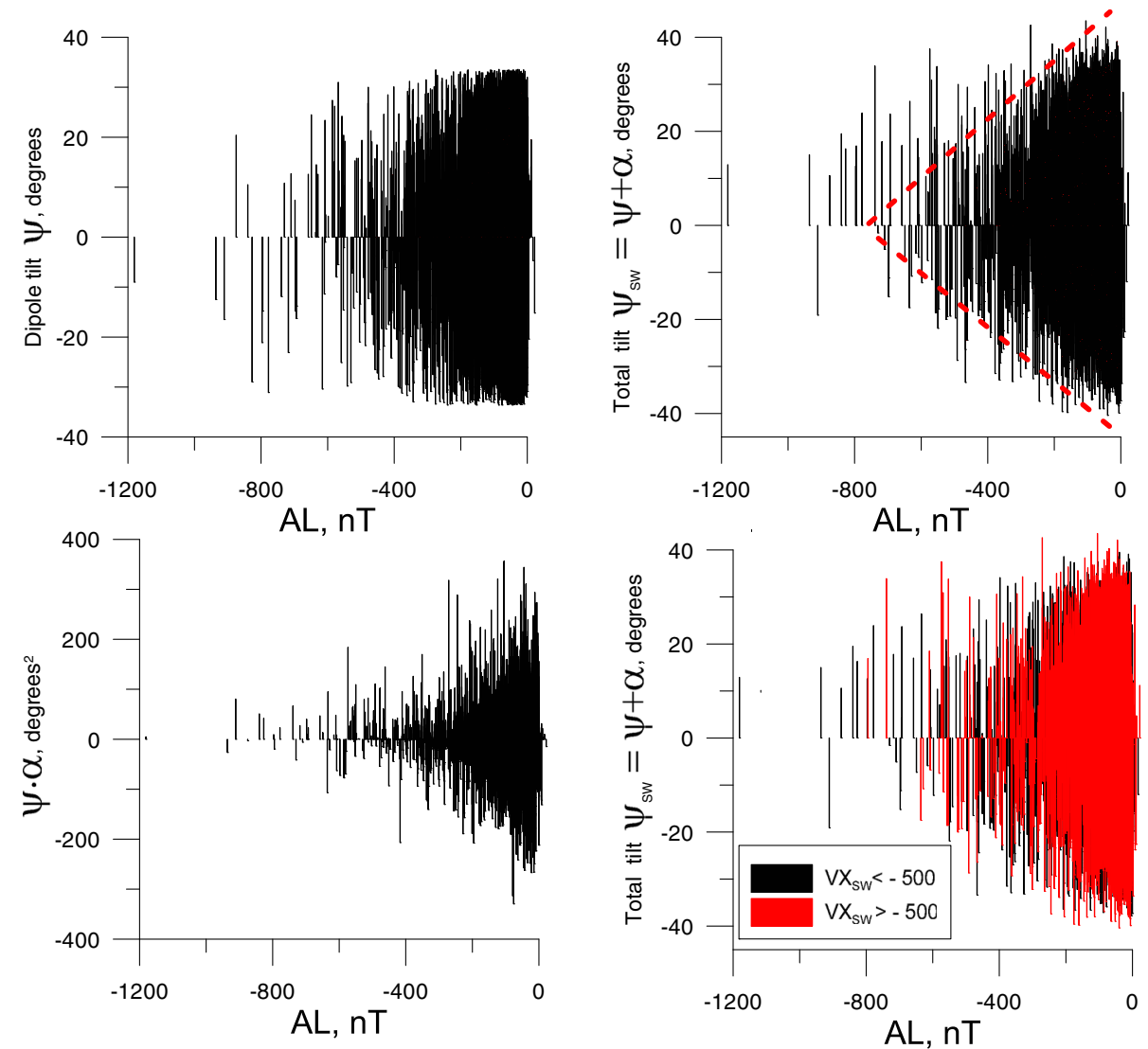

Fig. 6 The minimal substorm AL index (horizontal axis) versus tilt-related values (vertical bars) as follows: dipole tilt (top left panel), effective tilt (top right panel), product of the dipole tilt and SW inclination $\alpha$ (bottom left). Right bottom plot is essentially the same as the top right one, but it includes the substorms that occurred under SW flow velocities below $500 \mathrm{~km} / \mathrm{s}$, which are shown by red bars

bending and deflection from the Sun-Earth line. Since the observations of $B_{\text {lobe }}$ throughout the magnetosphere are essentially three-dimensional, we had to take into account the Y-variations of SW velocity and return to standard GSW coordinates with the $X_{\mathrm{GSW}}$-axis stretching in the anti-SW flow direction. The $Y_{\mathrm{GSW}}$-axis was defined to be perpendicular to the Earth's magnetic dipole so that the $X Z$-plane would contain the dipole axis.

\section{Method}

In order to check the above conjecture, we performed a modeling study of the spatial distribution of the tail lobe field and its response to the dipole tilt, and this effort was based on a large multi-year, multi-mission set of spacecraft data. The approach was to divide entire tail modeling region between $x=-10 R_{\mathrm{E}}$ and $x=-30 R_{\mathrm{E}}$ into a sequence of eight overlapping $5 R_{\mathrm{E}}$-wide bins of the $x$-coordinate, such that each bin spanned the interval $x_{i}-2.5 \leq x \leq x_{i}+2.5 R_{\mathrm{E}}$, where $x_{i}=-10-2.5 \cdot i$ with $i=1,2, \ldots, 8$. The spacecraft data were then sorted out into subsets of records falling within each of the eight distance bins, and eight local field models were generated corresponding to each interval.

An obvious advantage of this approach is that, owing to the limited spatial extent of the overlapping domains, the local magnetic field distribution can be modeled using relatively simple mathematical forms. A method of this kind was used in an earlier work by Peredo et al. (1993), where we modeled the field $\mathbf{B}$ by suitable expansions for a two-component vector potential $\mathbf{A}=A_{x} \mathbf{e}_{x}+A_{y} \mathbf{e}_{y}$, which automatically ensured the condition $\nabla \cdot \mathbf{B}=0$. In the early phase of the present work, a trial model of the same form was also constructed. However, it was then realized that, since we are primarily interested in knowing the local magnitude of the tail current, there is no need to model the full magnetic field vector $\mathbf{B}$. Rather, it is sufficient to develop a model representing either the scalar strength $B$ or the Sun-Earth component of the tail field $B_{x}$, whose jump across the current sheet is proportional to the local total current per unit tail length. Modeling only one scalar quantity instead of the full vector allowed 
us to reduce the number of unknown model parameters, which, in turn, made it possible to conveniently take into account the state of the interplanetary medium by splitting the expansion coefficients into two parts dependent on the SW pressure-related and an IMF-related driver function. In this study, we experimented with local models of both types (i.e., representing both $B$ and $B_{x}$ ), and both approaches yielded nearly the same results. A more detailed description of what was found from the $B_{x}$ modeling is given in the following sections.

\section{Data selection and binning}

A critical factor in these kinds of studies is the density and evenness of the data coverage of the modeling region in both geometric and parametric space. Since our ultimate object of interest is the distribution of the total current per unit tail length (i.e., the volume density $j$ integrated in the $z$-direction), each interval of the tailward distance should contain enough data taken both northward and southward from the current sheet. This study used a large multi-year set of space magnetometer data that were obtained by Geotail (1995-2013), Cluster (2001-2012), and five THEMIS probes (2007-2013), these data were taken tailward from the plane $x=-10 R_{\mathrm{E}}$. Figure 7 illustrates the data distribution in the eight intervals of $x$ in projection onto the $X Z$ and $X Y$-planes in the GSW coordinate system. In order to keep the models mathematically simple, the data were also restricted to limited ranges of $y$ - and $z$-coordinates, with the selection window size gradually widening down the tail, as shown in the plots. All the data had 5-min resolution, the number of records in the individual (overlapping) subsets varied from $\sim 32,000$ to $\sim 82,000$, and the total number of records used in this study was 264,552 . Each data record was provided with concurrent information on the interplanetary medium conditions, which were obtained from 5-min average yearly files with OMNI data.

\section{Model}

The model distribution of the tail $B_{x}$ was assumed in the form

$$
\begin{gathered}
B_{x}=B_{x, \text { dip }}+f_{1}\left(z-Z_{s}\right)\left[a_{1} P_{n}^{\gamma_{1}}+a_{2} P_{n}^{\gamma_{2}} \Psi^{2}+a_{3} F_{n}\right. \\
\left.+a_{4} F_{n} \Psi^{2}+a_{5} \Psi f_{1}\left(z-Z_{s}\right) P_{n}^{\gamma_{3}}\right] \\
+f_{2}\left(z-Z_{s}\right)\left[a_{6} P_{n}^{\gamma_{4}}+a_{7} P_{n}^{\gamma_{5}} \Psi^{2}+a_{8} F_{n}+a_{9} F_{n} \Psi^{2}\right. \\
\left.+a_{10} \Psi f_{2}\left(z-Z_{s}\right) P_{n}^{\gamma_{6}}\right]
\end{gathered}
$$

which includes the geodipole contribution $B_{x, \text { dip; }}$ the remaining terms represent the field of external sources. Here we used simple functions $f_{1}(z)=z /\left(z^{2}+D^{2}\right)^{1 / 2}$ and $f_{2}(z)=z /\left(z^{2}+D^{2}\right)$ to represent the local north-south profiles of $B_{x}(z)$. The first factor, $f_{1}$, is an odd smoothed step function which reverses its sign at $z=0$ and varies over a characteristic thickness $2 D$ between asymptotic limits $-1<f_{1}<+1$. The second factor, $f_{2}$, differs from $f_{1}$ only in that it asymptotically tends to zero at $z \rightarrow \pm \infty$, which allows for gradual north-south variation of $B_{x}$ in the lobes and thus lends more flexibility to the model. The half-thickness $D$ of the current sheet was assumed in each distance bin as a symmetric parabolic function of the normalized distance $y_{n}=y / 10$ across the tail: $D=D_{0}+D_{1} y_{n}^{2}$.

The bracketed terms in Eq. (1) quantify the response of the model $B_{x}$ to the SW ram pressure $P_{n}=P /\langle P\rangle$, which is normalized by its average value $\langle P\rangle=1.6 \mathrm{nPa}$, the driving parameter $F_{n}$ associated with the IMF, and the dipole tilt angle $\Psi$. The function $F_{n}=10^{-4} \cdot V_{\mathrm{SW}}^{4 / 3} B_{t}^{2 / 3} \sin ^{8 / 3}(\theta / 2)$ is an index by Newell et al. (2007), which is normalized by the value $F=10^{4}$ corresponding to the SW speed 450 $\mathrm{km} / \mathrm{s}$ and a purely southward IMF with $B_{z}=-5 \mathrm{nT}$.

The dipole tilt $\Psi$ controls both the magnitude of the tail current and its geometry; accordingly, the tilt effects enter in the model described by Eq. (1) in a twofold manner.
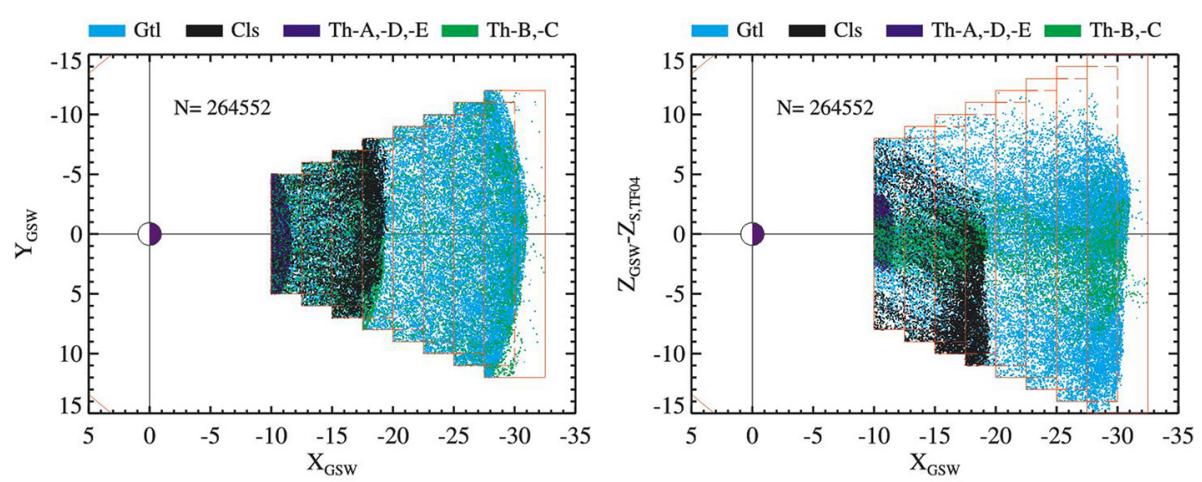

Fig. 7 Spacecraft data distribution in the sequence foe eight sliding bins of $X_{G S W}$ projected on the GSW equatorial (left) and meridional (right) planes 
The first mode of response is explicitly represented by the terms with the factors $\Psi$ and $\Psi^{2}$. Note here that both brackets in Eq. (1) include two types of terms that correspond to opposite symmetry properties with respect to $z$ and $\Psi$. Terms of the first type correspond to the overall decrease or increase of $\left|B_{x}\right|$ in both lobes and, as such, include only even powers of $\Psi$. The other type represents the north-south asymmetry induced by $\Psi \neq 0$, which implies only odd powers of $\Psi$. Owing to a general symmetry constraint on the model $B_{x}$, which requires that $B_{x}(x, y, z, \Psi)=-B_{x}(x, y,-z,-\Psi)$ (e.g., Mead and Fairfield 1975; Eq. (4)) terms that are odd with respect to $\Psi$ are even with respect to $z$ and vice versa.

The second mode of response to the dipole tilt is the warping of the magnetic configuration in both the $X Z$ - and $Y Z$-planes (e.g., Tsyganenko and Fairfield 2004; referred to henceforth as TF04). That effect was taken into account in Eq. (1) by replacing $z$ in $f_{1}$ and $f_{2}$ by $z-Z_{s}$, where $Z_{s}=Z_{s}(x, y, \Psi)$ is a function of the position and dipole tilt angle $\Psi$ specifying the tilt-dependent shape of the deformed current sheet. It was adopted in a form similar to that used earlier in TF04, with the exclusion of the IMF $B_{y}$-related twisting term, which was assumed to be small with respect to those due to the dipole tilt. This was done because we are mostly interested in effects close to the tail axis and at relatively close geocentric distances. Specifically, the north-south shift was represented as

$$
\begin{aligned}
Z_{s} & =-x \tan \Psi^{*}-G\left|y_{n}\right|^{3} \sin \Psi, \sin \Psi^{*} \\
& =\sin \Psi\left[1+\left(-x / R_{\mathrm{H}}\right)^{\alpha}\right]^{-1 / \alpha}
\end{aligned}
$$

where $R_{\mathrm{H}}$ is the hinging distance, $G$ is the transverse warping factor, and $\alpha$ is a parameter defining the sharpness of the kink in the tail current hinging area (see TF04 for more details). Based on previous results (TF04; Tsyganenko and Andreeva 2014), the hinging distance was further represented as a function of the interplanetary drivers

$$
R_{\mathrm{H}}=R_{\mathrm{H}_{0}} P_{n}^{\chi}+R_{\mathrm{H}_{1}} F_{n}
$$

where $R_{\mathrm{H}_{0}}, R_{\mathrm{H}_{1}}$, and $\chi$ are free model parameters. Since our region of interest lies tailward from the current sheet hinging area, the parameter $\alpha$ was fixed at a constant value of $\alpha=3$ based on previous estimates in TF04.

In total, the model Eqs. (1)-(3) contain 22 unknown free parameters including ten linear coefficients $a_{i}, i=$ $1, \ldots 10$ and 12 nonlinear parameters $D_{0}, D_{1}, R_{H_{0}}, R_{H_{1}}$, $\chi, G$, and $\gamma_{1}-\gamma_{6}$. Their values were derived for each distance bin using a search code based on the downhill simplex Nelder-Mead method, combined with a singular value decomposition (SVD) algorithm (Press et al. 1992) to calculate the linear coefficients at each simplex step.

\section{Model results}

Figure 8 shows distributions of the absolute value $\left|B_{x}\right|$ in four cross-tail sections, each bounded within its corresponding data selection window in the $y$-and $z$-directions. All the plots correspond to the dipole tilt angle $\Psi=30^{\circ}$, SW pressure $P=3 \mathrm{nPa}$, and the IMF driver $F_{n}=1$. In the midnight meridian plane, the current sheet center is located around $Z_{s} \approx 4 R_{\mathrm{E}}$, which is consistent with the best-fit value of the hinging distance of $R_{\mathrm{H}} \approx 8 R_{\mathrm{E}}$. Both the absolute magnitude $\left|B_{x}\right|$ and its north-south asymmetry are greatest in the nearest distance bin and then gradually decrease tailward (note the different range of the color bars in the successive plots).

This agrees with an earlier finding (Tsyganenko 1998, Fig 4) based on a much smaller dataset, as well as with the result of a magnetohydrodynamic (MHD) simulation run,which is presented next in Fig. 9. To check if similar magnetic field behavior with dipole tilt was produced by global MHD modeling, a set of global MHD simulations with synthetic SW input was performed through the Community Coordinated Modeling Center in the Goddard Space Flight Center (http://ccmc.gsfc.nasa.gov/). Here, we present the results of one of this simulation that was made using Block-Adaptive-Tree-SolarwindRoe-Upwind-Scheme (BATSRUS) model (Powell et al. 1999). This simulation was performed in a numerical grid with 1 million cells [minimal grid size is 0.25 Earth radii $\left(R_{\mathrm{E}}\right)$ in the inner magnetosphere, $0.5 R_{\mathrm{E}}$ in the dayside regions of the magnetopause and bow-shock, in mid-tail plasma sheet, and $1 R_{\mathrm{E}}$ in the mid-tail magnetopause and lobes]. The simulation had a duration of $30 \mathrm{~min}$ (in addition to $1 \mathrm{~h}$ of preconditioning) with fixed SW input in order to obtain the quasi-stationary solution. Namely, the $\mathrm{SW}$ speed $V_{x}=-400 \mathrm{~km} / \mathrm{s}, V_{y}=V_{z}=0$, proton temperature $T=105 \mathrm{~K}$, number density $N=5 \mathrm{~cm}^{-3}$, and IMF $\left(B_{x}, B_{y}, B_{z}\right)=(0,0,-4) \mathrm{nT}$. Ionospheric conductance was also fixed with uniform Hall and Pedersen components. The dipole tilt was set to $\Psi=+30$ (North Pole tilted towards the Sun) without updating.

Based on the results of fitting the model Eq. (1) to the data subsets for the eight sliding bins of $x$ - coordinates shown in Fig. 7, we calculated for each interval $\Delta B_{x}=$ $0.5\left(B_{x}^{(N)}-B_{x}^{(S)}\right)$, where $B_{x}^{(N)}$ and $B_{x}^{(S)}$ correspond to the midnight locations at $z=Z_{s} \pm 3 R_{\mathrm{E}}$ northward and southward of the current sheet center. The plots of $\Delta B_{x}$ shown in Fig. 10 represent the variation of the average tail lobe field (and thereby the current) along the Sun-Earth line in the interval $-30 \leq x \leq-10 R_{\mathrm{E}}$ for four combinations of the SW pressure $P$ and the IMF parameter $F$. Each panel shows two profiles, which were calculated for the case of a planar current sheet ( $\Psi=0$, green solid line) and a warped current sheet ( $\Psi=30^{\circ}$, black solid line). Red and blue dashed lines show, respectively, the southern and northern lobe fields $-B_{x}^{(S)}$ and $B_{x}^{(N)}$ entering in the 

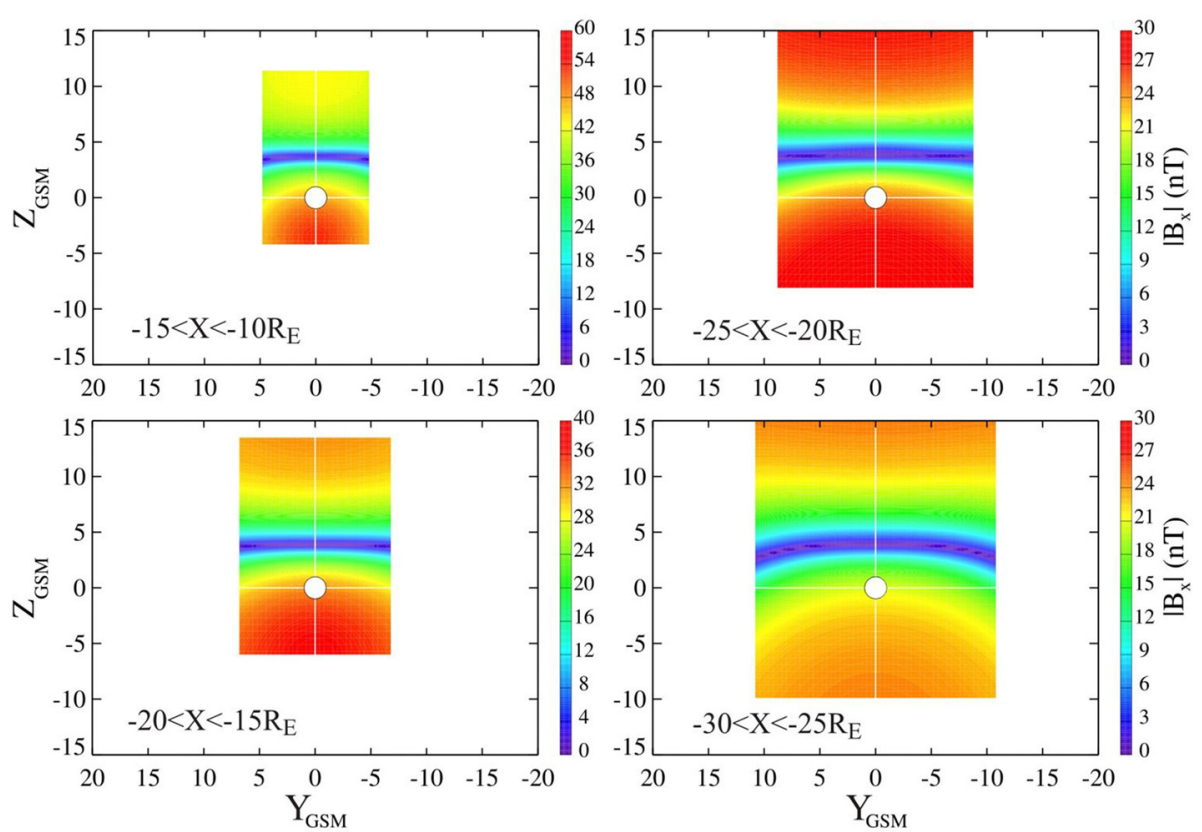

Fig. 8 Cross-tail distributions of the model $\left|B_{x}\right|$ for four intervals of $x$-coordinates corresponding to the largest dipole tilt $\Psi=30^{\circ}$. The height and width of the plots correspond to those of the data selection windows. Note the different ranges for the color scale bars in the individual panels

half-sum $\Delta B_{x}$ for the tilted case $\Psi=30^{\circ}$. Error bars in the plots correspond to the values of the residual root mean square (rms) difference between the observed and model fields for each distance bin. As expected, in all four cases, the field decreases downtail almost monotonically, except in the farthest distance bins beyond $25 R_{\mathrm{E}}$. The principal effects of interplanetary conditions are also clearly seen, which involve a significant increase of the tail field with growing ram pressure $P$ and the IMF index $F$, in agreement with earlier results (e.g., Fairfield and Jones 1996; Tsyganenko 2000).

In the context of the present study, the most interesting feature is a distinct overall decrease of the tail current in the tilted case; in all four plots, the black line is positioned significantly lower than the green line. The largest relative difference (nearly $25 \%$ ) was observed in the three nearest bins of $x$ for the case with $P=4 \mathrm{nPa}$ and $F_{n}=0$.

\section{Overall results and discussion}

In this study, we statistically examined the conjecture that the threshold of substorm initiating instabilities (of any possible origin) may depend on the curvature of the nightside magnetospheric current sheet, which is, in turn, determined by the dipole tilt angle in the GSW coordinate system, as defined by the mutual orientation of the geodipole axis and the SW flow direction. Two independent statistical and modeling approaches were applied, and both of them led us to the conclusions that
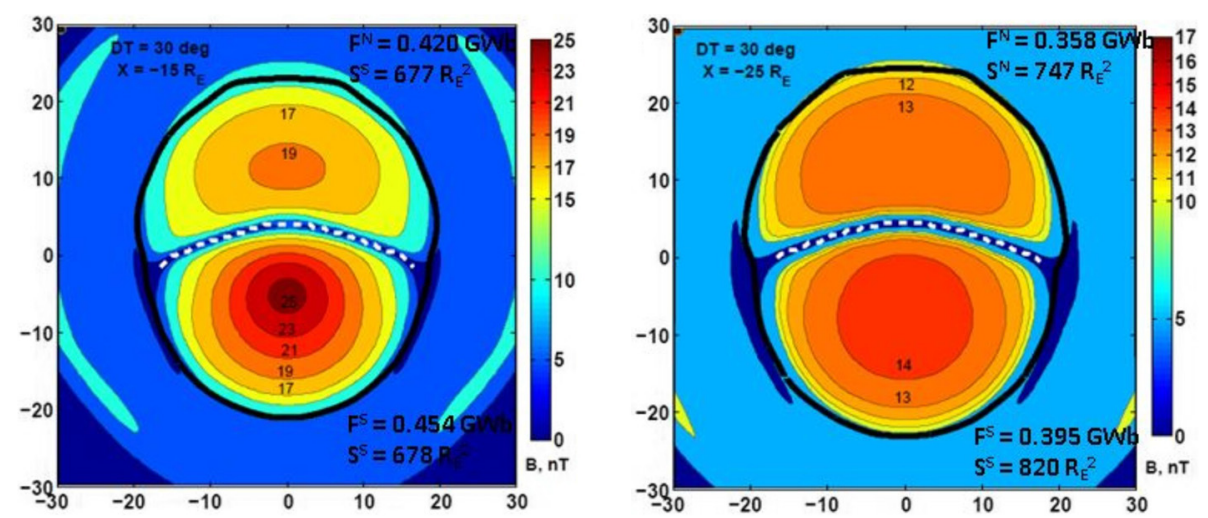

Fig. 9 Illustration of the asymmetry of the tail lobe field associated with the dipole tilt; data are based on an MHD simulation run with $\Psi=30^{\circ}$ 

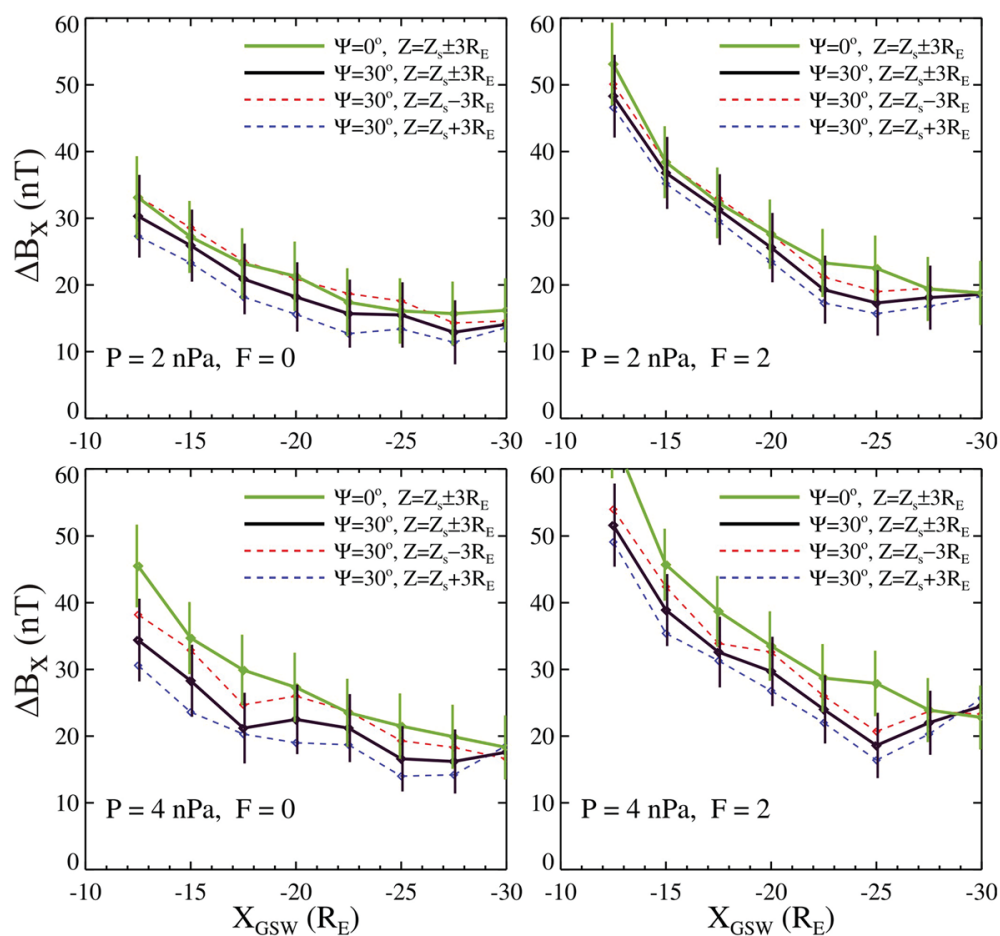

Fig. 10 Distributions of the model $\Delta B_{X}=0.5\left(B_{x}^{(N)}-B_{X}^{(S)}\right)$ (proportional to the current per tail unit length) along the $x$-axis for four combinations of the interplanetary driving parameters. Black and green solid lines correspond to the tilted $\left(\Psi=30^{\circ}\right)$ and perpendicular $(\Psi=0)$ orientation of the geodipole, respectively. Red and blue dashed lines show the southern and northern lobe field variation, respectively

at larger tilt angles, substorms set off at lower energy input and, conversely, more symmetric current sheets at smaller tilt angles are more effective in storing the magnetic energy and more stable against substorm initiating instabilities.

Summarizing the results, we put forth the following assertions:

1. The substorm probability is $10-25 \%$ larger during the periods with tilts above $16^{\circ}$, in comparison with the periods with smaller tilts. This point is probably the weakest of all, because even though we clearly see an increase of the substorm probability for most of the tilt ranges in Fig. 3, there exists a significant drop in two tilt angle bins immediately adjacent to the largest $|\Psi|$ bins in the histogram. Additionally, we found largely different probabilities for negative and positive tilts. Thus, although the overall dependence confirms the above statement, there are some additional factors that call for further investigation. One of them is the possible influence of unevenness of data in the set, as reflected in the fact that the number of substorms from the Southern Hemisphere is only half of that in the Northern Hemisphere, in spite of the data being selected from a twofold larger time interval.
2. Substorm probability is larger by $10-20 \%$ if the SW flow direction increases the dipole tilt computed in the standard GSM coordinate system. This tendency is clear for all tilt ranges, except the smallest near zero tilt.

3. The intensity of a substorm, if quantified by the minimum AL value, is smaller for the periods with larger tilts. The effect is better seen if we operate with the effective tilt $\Psi_{\mathrm{SW}}$, and provided that the fast flows are excluded from the analysis. It is worth noting that a very similar result-a larger activity for smaller dipole tilts-was obtained by Nowada et al. (2009) for the period 1978-1988 for both the AL and $\mathrm{AU}$ indices (with a larger effect for $\mathrm{AL}$ ), and it is best seen during intervals with negative IMF $B_{z}$.

4. The overall statistic for the $B_{\text {lobe }}$ distribution based on empirical modeling shows that the average lobe field is smaller for larger tilts at all distances within a wide range of the SW parameters. The difference in $B_{\text {lobe }}$ between small and large tilts varies within 10-20\% depending on the radial distance.

\section{Conclusions}

A new feature of our approach is that in analyzing the current sheet shape, we used the tilt angles modified by 
the SW flow direction and found that the relatively small modification of the standard $\Psi_{\mathrm{GSM}}$ tilt due to taking into account the SW flow direction proved to be rather important. A possible reason for the importance of including the SW direction may be its rapid changes, which are much faster than the relatively slow rotation of the geodipole axis.

Our final conclusion involving the importance of the shape of the current sheet for the substorm dynamics calls for further investigation. Specifically, we need to understand what features of the deformed current sheet play a dominant role in substorm initiation. Kivelson and Hughes (1990) suggested that excessive field line curvature was the most important factor for the substorm instability threshold. We also point out that there may be a possible role for the north-south asymmetry of $B_{\text {lobe }}$ values due to large dipole tilts. Additionally, fast flows that originate in asymmetric and curved magnetic configurations may be an important factor in creating the substorm current wedge system and in the ensuing dipolarization process.

\section{Competing interests}

The authors declare that they have no competing interests.

\section{Authors' contributions}

All authors read and approved the final manuscript.

\section{Acknowledgements}

This work was supported by the Russian Science Foundation (grant no. 1417-00072). We thank V. Sergeev for valuable comments and fruitful discussions.

\section{Author details}

${ }^{1}$ Saint Petersburg State University, St. Petersburg, Russia. ${ }^{2}$ Finnish Meteorological Intitute, Helsinki, Finland; currently at University Centre in Svalbard, Longyearbyen, Norway.

Received: 24 March 2015 Accepted: 11 August 2015

Published: 28 August 2015

\section{References}

Fairfield DH, Jones J (1996) Variability of the tail lobe field strength. J Geophys Res 101:7785-7791

Ganushkina NYu, Kubyshkina MV, Partamies N, Tanskanen E (2013) Interhemispheric magnetic conjugacy. J Geophys Res 118:1049-1061. doi:10.1002/jgra.50137

Juusola L, Ostgaard N, Tanskanen E, Partamies N, Snekvik K (2011) Earthward plasma sheet flows during substorm phases. J Geophys Res 116:A10228. doi:10.1029/2011JA016852

Kissinger J, McPherron RL, Hsu TS, Angelopoulos V (2011) Steady magnetospheric convection and stream interfaces: relationship over a solar cycle. J Geophys Res 116:A00119. doi:10.1029/2010JA015763

Kivelson MG, Hughes WJ (1990) On the threshold for triggering substorms. Planet Space Sci 38:211-220

Kubyshkina M, Sergeev V, Tsyganenko N, Angelopoulos V, Runov A, Donovan E, Singer H, Auster U, Baumjohann W (2011) Time-dependent magnetospheric configuration and breakup mapping during a substorm. J Geophys Res 116:A00I27. doi:10.1029/2010JA015882

Mead GD, Fairfield DH (1975) A quantitative magnetospheric field model derived from spacecraft magnetometer data. J Geophys Res 80:523-534

Newell PH, Sotirelis T, Liou K, Meng Cl, Rich FJ (2007) A nearly universal solar wind-magnetosphere coupling function inferred from 10 magnetospheric state variables. J Geophys Res 112:A01206. doi:10.1029/2006JA012015
Nowada M, Shue JH, Russell CT (2009) Effects of dipole tilt angle on geomagnetic activity. Planet Space Sci 57:1254-1259

Panov EV, Nakamura R, Baumjohann W, Kubyshkina MV, Artemyev AV, Sergeev VA, Petrukovich AA, Angelopoulos V, Glassmeier KH, McFadden JP, Larson D (2012) Kinetic ballooning/interchange instability in a bent plasma sheet. J Geophys Res 117:A06228. doi:10.1029/2011JA017496

Partamies N, Pulkkinen TI, McPherron RL, McWilliams K, Bryant CR, Tanskanen E, Singer HJ, Reeves GD, Thomsen MF (2009) Different magnetospheric modes: solar wind driving and coupling efficiency. Ann Geophys 27:4281-4291

Partamies N, Juusola L, Tanskanen E, Kauristie K (2013) Statistical properties of substorms during different storm and solar cycle phases. Ann Geophys 31:349-358. doi:10.5194/angeo-31-349-2013

Peredo M, Stern DP, Tsyganenko NA (1993) Are existing magnetospheric models excessively stretched? J Geophys Res 98:15343-15354

Powell KG, Roe PL, Linde TJ, Gombosi TI, De Zeeuw DL (1999) A solution-adaptive upwind scheme for ideal magnetohydrodynamics. J Comput Phys 154:284-309. doi:10.1006/jcph.1999.6

Press WH, Teukolsky SA, Vetterling WT, Flannery BP (1992) Numerical recipes in Fortran. 2nd ed. Cambridge Univ. Press, New York

Sergeev V, Angelopoulos V, Kubyshkina M, Donovan E, Zhou XZ, Runov A, Singer H, McFadden J, Nakamura R (2011) Substorm growth and expansion onset as observed with ideal ground-spacecraft THEMIS coverage. J Geophys Res 116:A00126. doi:10.1029/2010JA015689

Tsyganenko NA (1998) Modeling of twisted/warped magnetospheric configurations using the general deformation method. J Geophys Res 103:23551-23563

Tsyganenko NA (2000) Solar wind control of the tail lobe magnetic field as deduced from Geotail, AMPTE/IRM, and ISEE 2 data. J Geophys Res 105:5517-5528

Tsyganenko NA, Fairfield DH (2004) Global shape of the magnetotail current sheet as derived from Geotail and Polar data. J Geophys Res 109:A03218. doi:10.1029/2003JA010062

Tsyganenko NA, Andreeva VA (2014) On the "bowl-shaped" deformation of planetary equatorial current sheets. Geophys Res Lett 41:1079-1084. doi:10.1002/2014GL059295

Tsyganenko NA, Andreeva VA, Gordeev El (2015) Internally and externally induced deformations of the magnetospheric equatorial current as inferred from spacecraft data. Ann Geophys 33:1-11. doi:10.5194/angeo-33-1-2015

\section{Submit your manuscript to a SpringerOpen ${ }^{\circ}$ journal and benefit from:}

- Convenient online submission

- Rigorous peer review

- Immediate publication on acceptance

- Open access: articles freely available online

- High visibility within the field

- Retaining the copyright to your article

Submit your next manuscript at $\gg$ springeropen.com 